\title{
Field Dependence of the Critical Current and Its Relation to the Anisotropy of BSCCO Conductors and Coils
}

\author{
H. W. Weijers, B. ten Haken, H. H. J. ten Kate, and J. Schwartz, Fellow, IEEE
}

\begin{abstract}
The design of HTS magnets is often based on the properties of a number of short samples that are presumed to be representative of the conductor to be used. Variability in conductor properties and inhomogeneity in the magnetic field distribution within the magnets, coupled with conductor anisotropy, provide a significant challenge to accurately predict the field dependence of the magnet critical current. This work is based on measured superconducting properties of $\mathrm{Bi}-2212$ and $\mathrm{Bi}-2223$ conductors at 4.2 $\mathrm{K}$ in parallel and perpendicular magnetic fields up to $33 \mathrm{~T}$. Properties of double pancake units and stacks, from the same or similar conductor batches, are presented, based on measurements at self-field and in applied co-axial background magnetic fields up to 19 T. Modeling of this data is based on short sample properties in perpendicular field; the average grain misalignment is used as the parameter to quantify the anisotropy. Correlations and discrepancies between the measured data and models based on short sample data are discussed for $\mathrm{Bi}-2212$ and $\mathrm{Bi}-2223$ conductors.
\end{abstract}

Index Terms-Anisotropy, coils, critical current, field dependence.

\section{INTRODUCTION}

$\mathbf{T}$ HE ABILITY to predict the critical current of coils based on the properties of its constituent conductor is essential in magnet design. The relation between conductor and coil $I_{\mathrm{c}}$ is more complicated for HTS conductors than metallic superconductors due to the strong anisotropy typically observed in practical HTS conductors. Despite significant advances in the development of $\mathrm{BiSrCaCuO}$ superconductors without anisotropy at the conductor level [1], [2], anisotropic tape conductors remain widely used in HTS magnet technology [3], [4]. A desire to understand and predict the properties of HTS sections of $25 \mathrm{~T}$ superconducting magnets provided the motivation for this work. Specifically, the self-field critical current $I_{\mathrm{c}}$ (self) of double pancakes is hard to predict, but is essential for magnets consisting of double pancakes. Relations between $I_{\mathrm{c}}$ of short samples, double pancakes and larger coils must be developed so qualification standards and design procedures can be developed.

Manuscript received October 4, 2004. This research is supported in part by the NSF and the State of Florida under cooperative grant DMR 0084173.

H. W. Weijers is with the National High Magnetic Field Laboratory, Tallahassee, FL 32310 USA (e-mail: weijers@ magnet.fsu.edu).

B. ten Haken and H. H. J. ten Kate are with the Faculty of Science and Technology, University of Twente, 7500 AE Enschede, The Netherlands (e-mail: B.tenHaken@tnw.utwente.nl; h.h.j.tenkate@tnw.utwente.nl).

J. Schwartz is with the National High Magnetic Field Laboratory, Tallahassee, Fl 32310 USA. He is also with the Dept. of ME, FAMU-FSU College of Engineering, Tallahassee, FL 32310 USA (e-mail: schwartz@magnet.fsu.edu).

Digital Object Identifier 10.1109/TASC.2005.847650
TABLE I

CONDUCTOR AND MODEL PARAMETERS

\begin{tabular}{lll}
\hline \hline & Conductor A & Conductor B \\
\hline Material & Bi-2212 & Bi-2223 \\
Conductor width [mm] & 4.95 & 2.92 \\
Conductor thickness [mm] & 0.195 & 0.243 \\
$I_{\mathrm{c} \_s s}$ (self) [A] & 512 & 578 \\
$n$-value & 8 & 19 \\
$I_{\mathrm{cs}}[\mathrm{A}]$ & 200 & 222 \\
$B_{\mathrm{sc}}[\mathrm{T}]$ & 54 & 97 \\
$I_{\mathrm{cw}}[\mathrm{T}]$ & 445 & 608 \\
$B_{\mathrm{O}}[\mathrm{T}]$ & 0.22 & 0.09 \\
\hline
\end{tabular}

The anisotropy in $I_{\mathrm{c}}(\boldsymbol{B})$ for both a $\mathrm{Bi}_{2} \mathrm{Sr}_{2} \mathrm{CaCu}_{2} \mathrm{O}_{\mathrm{x}}$ (Bi2212) and a $\mathrm{Bi}_{2} \mathrm{Sr}_{2} \mathrm{Ca}_{2} \mathrm{Cu}_{3} \mathrm{O}_{\mathrm{x}}$ (Bi-2223) conductor is quantified with a single conductor-specific parameter: the average grain misalignment $\alpha^{*}$ [5]-[7]. Use of this parameter reduces the vector relation $I_{\mathrm{c}}(\boldsymbol{B})$ to a scalar relation $I_{\mathrm{c}}\left(\boldsymbol{B}_{\mathrm{eff}}\right)$, where $\boldsymbol{B}_{\text {eff }}$ is the effective field perpendicular to the conductor resulting in the same $I_{\mathrm{c}}$ as the vector $\boldsymbol{B}$ at angle $\phi$. Following the references, $\boldsymbol{B}_{\text {eff }}$ is calculated from the total field value $B_{\text {total }}$ and a scaling function $f(\phi)$ that is unique for each value of $\alpha^{*}$ :

$$
\boldsymbol{B}_{\text {eff }}=B_{\text {total }} \cdot f(\phi)
$$

This work relates measured field dependence of coils to short sample properties using (1) to model anisotropy.

\section{CONDUCTORS}

The conductors considered here are reacted PIT multifilamentary tapes. The $I_{\mathrm{c}}$ measurements in perpendicular and parallel magnetic fields at $4.2 \mathrm{~K}$ are described elsewhere [8]. The model used for $I_{\mathrm{c}}(B)$ for perpendicular field assumes parallel networks of strongly and weakly linked paths, contributing $I_{\mathrm{cs}}$ and $I_{\mathrm{cw}}$ to $I_{\mathrm{c}}$ in self-field conditions, respectively, and whose field dependence is in turn characterized with parameters $B_{\mathrm{sc}}$ and $B_{\mathrm{o}}$. Several different models exist to describe $I_{\mathrm{C}}(B)$ based on various physical phenomena and models. Here however, it suffices to note that for applied fields over $0.1 \mathrm{~T}$ the measurements are described, using (2) and the parameters of Table II, with an average error below $1 \%$. For low values of $B$, (2) results in calculated values larger than the measured short sample self-field value $I_{\mathrm{C}_{-} \mathrm{SS}}($ self $)$. In those cases $I_{\mathrm{C}}$ is taken to be $I_{\mathrm{C} \_ \text {SS }}($ self $)$. It is assumed that the normalized $I_{\mathrm{c}}(B)$ 
TABLE II

GEOMETRIC DOUBLE-PANCAKE PARAMETERS

\begin{tabular}{llll}
\hline \hline Symbol & Unit & Coil A & Coil B \\
\hline Inner diameter & $\mathrm{mm}$ & 41 & 97.6 \\
Outer diameter & $\mathrm{mm}$ & 98 & 127 \\
Height & $\mathrm{mm}$ & 11 & 6 \\
Turns [-] & - & $2 \times 115$ & $2 \times 49$ \\
\hline
\end{tabular}

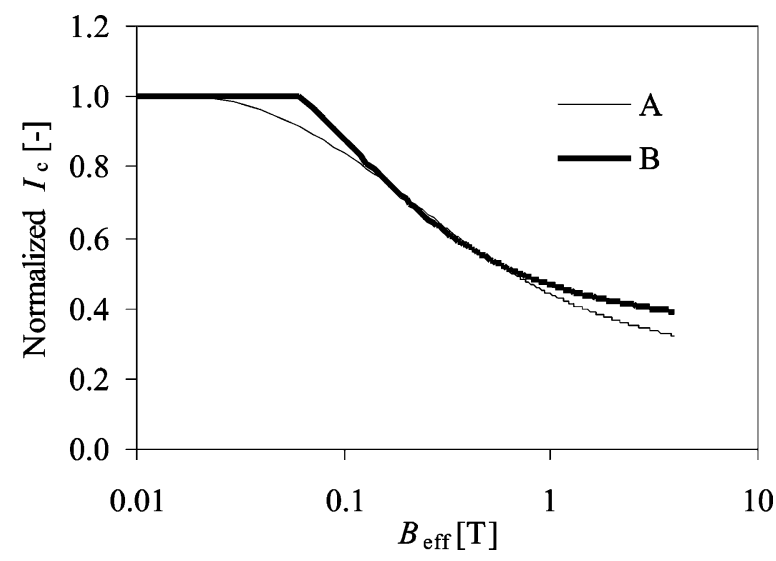

Fig. 1. Modeled normalized $I_{\mathrm{c}}(B)$ of conductors A and B.

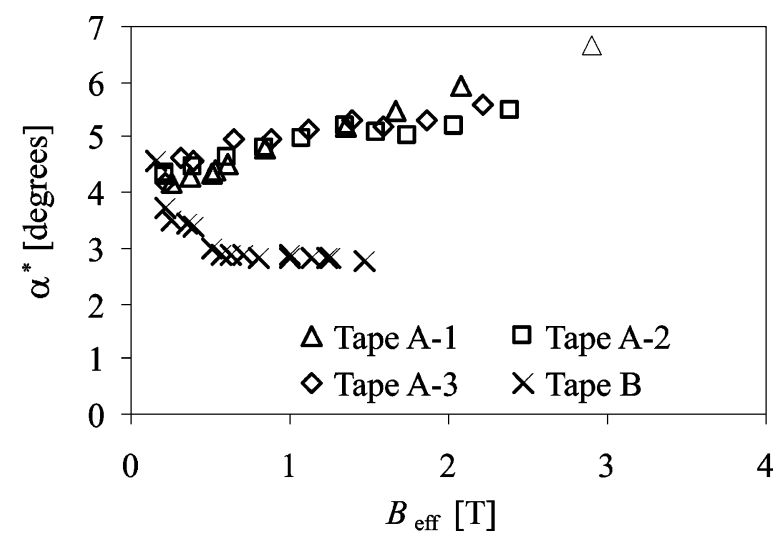

Fig. 2. Calculated $\alpha^{*}$ values for conductors A and B.

behavior, shown in Fig. 1, is identical for conductors with different $I_{\mathrm{c}}$ (self).

$$
I_{\mathrm{c}}(B)=\frac{I_{\mathrm{cw}}}{1+\frac{B}{B_{0}}}+I_{\mathrm{cs}} \exp \left(\frac{-B}{B_{\mathrm{sc}}}\right)
$$

The average grain misalignment $\alpha^{*}$ is determined from the ratio of parallel and perpendicular field resulting in the same $I_{\mathrm{C}}$, and is shown in Fig. 2 using [8]. Tapes A-1, A-2 and A-3 represent samples of conductor $\mathrm{A}$; tape $\mathrm{B}$ is a $\mathrm{Bi}-2223$ sample. For conductor $\mathrm{B}\left(B_{\mathrm{eff}}>0.5 \mathrm{~T}\right)$ the calculated value of $\alpha^{*}$ is fairly constant. The calculated value of $\alpha^{*}$ for conductor A appears to show a dependence on $B$, in contradiction with the anisotropy model, although the variation is within the margin of error. (a)

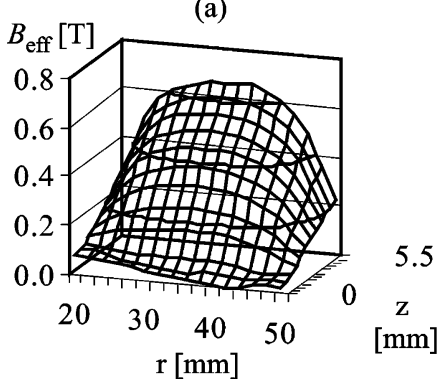

(b) $B_{\text {eff }}[\mathrm{T}]$

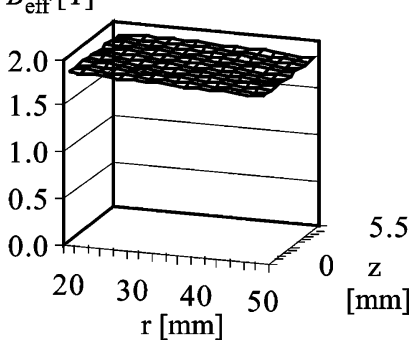

Fig. 3. Typical calculated values for $\boldsymbol{B}_{\text {eff }}$ in Coil A in the self-field condition (left) and in a $19 \mathrm{~T}$ background (right).

\section{DOUBLE PANCAKES}

\section{A. Measured Properties}

Two double pancake coils are discussed here. Both are epoxyimpregnated and produced from reacted conductor nominally identical to conductors A and B. Geometric parameters are listed in Table II. These coils were selected because their $I_{\mathrm{c}}(B)$ measurement is reversible within $1 \mathrm{~A}$, verifying the absence of stress induced $I_{\mathrm{c}}$ degradation. The measured properties are shown in the next section together with the model calculations.

\section{B. Model}

Quarter cross-sections of the windings are divided in 15 radial and 12 axial segments. The following parameters are calculated in the center of each segment: radial field, axial field, $B_{\text {total }}$, $\phi, \boldsymbol{B}_{\text {eff }}$, normalized $I_{\mathrm{c}}\left(\% I_{\mathrm{c}}\right)$, conductor length $l$ within the segment, electric field and voltage across the conductor length. The field components are calculated using the actual dimensions and current densities in the double pancakes and background magnet. The latter is described in [6]. The electric field $E$ along the conductor is calculated using

$$
E=E_{c \times} \times\left(\frac{I}{I_{c \_S S}(\operatorname{sel} f) \times \% I_{c}}\right)^{n}
$$

where $E_{\mathrm{c}}$ is the $10^{-4} \mathrm{~V} / \mathrm{m}$ criterion, $I$ the operating current, $I_{\text {C_SS }}$ the assumed short-sample conductor self-field $I_{\mathrm{c}}$ and $n$ the index of transition.

The most complicated condition to model for double pancakes is at self-field. The variations in magnetic field strength and direction are large at self-field and for low applied fields and the slope of $I_{\mathrm{c}}(B)$ is relatively steep. In contrast, for $B_{\text {applied }}>$ $5 \mathrm{~T}$ the field variations become relatively small and $I_{\mathrm{c}}$ is less sensitive to incremental changes in $\boldsymbol{B}$. Figs. 1, 3 and 6 illustrate this. Therefore, one may expect that a proper model accurately describes the field dependence of $I_{\mathrm{c}}$ for $B>5 \mathrm{~T}$, where $\boldsymbol{B}_{\text {eff }}$ is fairly homogeneous.

In the regime of $B_{\text {applied }}<5 \mathrm{~T}, \boldsymbol{B}_{\text {eff }}$ varies with radius and more so with $z$, i.e. across the width of a single turn. Fig. 3 illustrates that for Coil A. The axial variation of $\boldsymbol{B}_{\text {eff }}$ will promote an axial redistribution of current toward the midplane, where $\boldsymbol{B}_{\text {eff }}$ is lowest. This effect is approximated by introducing an axial variation of the current density $J=J(z)$, independent of radius. The axial variation of $J$ is determined iteratively such that the integral of electric field along the entire conductor length is 


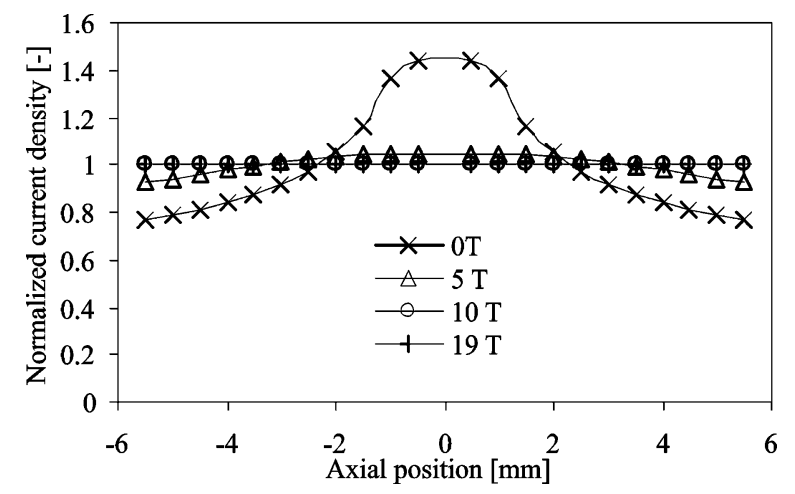

Fig. 4. Typical axial variation of current density within a turn versus applied field. Lines are a guide to the eye only.

TABLE III

COIL A FIT PARAMETERS

\begin{tabular}{lllll}
\hline \hline Current density & $\begin{array}{l}I_{\mathrm{c} \text { SS }}(\mathrm{self}) \\
{[\mathrm{A}]}\end{array}$ & $\alpha^{*}$ & $\begin{array}{l}n- \\
\text { value }\end{array}$ & $\begin{array}{l}\text { Average } \\
\text { error }\end{array}$ \\
\hline Constant & 302 & 6 & 8 & $3.8 \%$ \\
Axially variable & 296 & 4 & 6 & $3.8 \%$ \\
Axially variable & 302 & 6 & 6 & $2.9 \%$ \\
Axially variable & 319 & 8 & 6 & $3.8 \%$ \\
Axially variable & 296 & $4.06+0.071 \cdot B_{\text {total }}$ & 6 & $1.0 \%$ \\
Axially variable & 296 & $4.06+0.071 \cdot B_{\text {total }}$ & 8 & $0.9 \%$ \\
Axially variable & 296 & $4.06+0.071 \cdot B_{\text {total }}$ & 10 & $1.1 \%$ \\
\hline
\end{tabular}

independent of $z$. This variation, and its effect on $I_{\mathrm{c}}$, is significant below $10 \mathrm{~T}$. Typical behavior is shown in Fig. 4. The effect of this current redistribution on the local magnetic field strength and angle is taken into account.

Imperfect scaling for Bi-2212 samples [8] leaves doubts whether they can be modeled with a single, field independent value of $\alpha^{*}$. Therefore, $\alpha^{*}$ is alternatively set as a constant or described by

$$
\alpha^{*}=4.06+0.071 \cdot B_{\text {total }}
$$

Equation (4) is a linear fit to the $\alpha^{*}$ data of Fig. 2 when plotted versus applied parallel field. Thus $\alpha^{*}$ is calculated in every segment, and the scaling function $f(\alpha)$ follows from the average $\alpha^{*}$.

\section{Procedure}

For any given choice of $\alpha^{*}$ and $n$-value, a value for $I_{\mathrm{c}}(\mathrm{self})$ is chosen such that the calculated voltage across the coil corresponds to the $10^{-4} \mathrm{~V} / \mathrm{m}$ criterion when the operating current equals the measured critical current at $B_{\text {applied }}=19 \mathrm{~T}$. All free parameters are now determined. The operating current corresponding to $I_{\mathrm{c}}$ is then calculated for lower values of the background field while optimizing the axial current distribution to minimize the coil voltage.

\section{RESULTS}

\section{A. Bi-2212 Double Pancake}

Table III lists seven parameter sets used to model the measured $I_{\mathrm{C}}$ of Coil A and Fig. 5 shows the results. A comparison of the curves with and without axial grading (square and - markers)

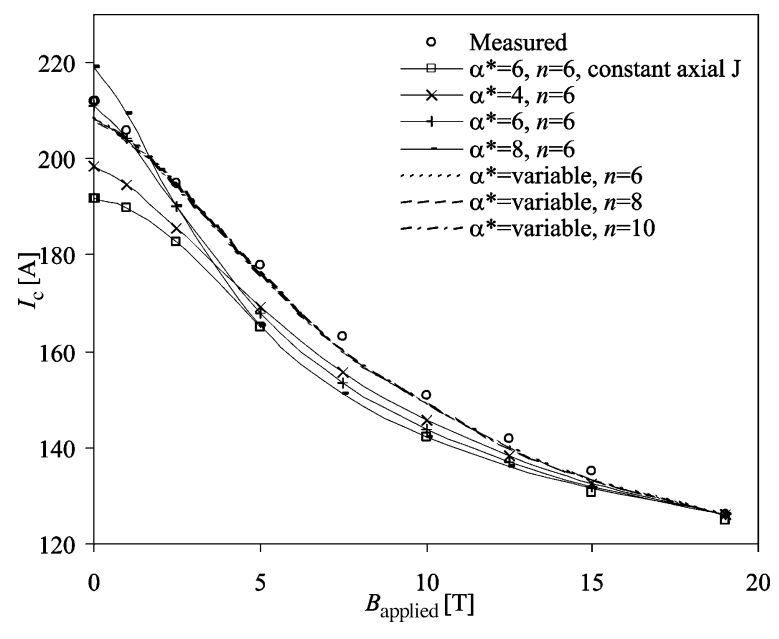

Fig. 5. Measurement and calculations of $\mathrm{I}_{\mathrm{c}}$ for Coil A. The curves for variable $\alpha^{*}$ are on top of each other.

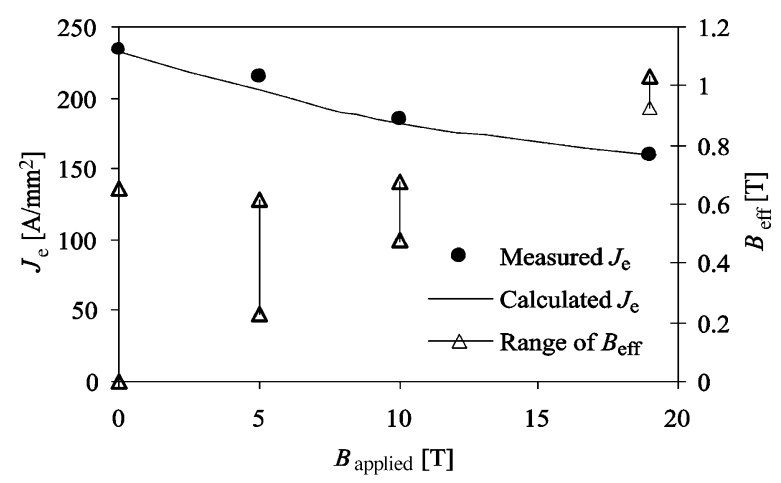

Fig. 6. Measurement and calculations of $I_{\mathrm{c}}$ for Coil B. The range between minimum and maximum $B_{\text {eff }}$ in the windings is on the right axis.

for $\alpha^{*}=6$ illustrates that axial grading only affects the results significantly for $B_{\text {applied }} \leq 5 \mathrm{~T}$. Axial grading improves the fit in that range.

Choosing various field independent values for $\alpha^{*}$ change the shape of the $I_{\mathrm{c}}(B)$ curve over the entire range, and most significantly for $B_{\text {applied }} \leq 5 \mathrm{~T}$. However, no value of $\alpha^{*}$ matches the slope of the $I_{\mathrm{c}}(B)$ curve for $B_{\text {applied }}>10 \mathrm{~T}$, nor for most of the lower field range.

Assuming the short sample data suggest a field dependence of $\alpha^{*}$ as in (4) results in a much closer fit to the measured data and is also practically independent of the $n$-value for $6<n<10$. The average fit error in coil $I_{\mathrm{c}}(B)$ is now comparable to the conductor $I_{\mathrm{c}}(B)$ fit error.

The calculated values for $I_{\mathrm{C}_{-} S S}$ (self) are significantly below the measured values for short samples cut from the same conductor batch as the coil; six samples show an average $I_{\mathrm{c}}$ of $403 \mathrm{~A}$ with a standard deviation of $7 \mathrm{~A}$. This is not satisfactory for predicting double pancake $I_{\mathrm{c}}$ from short sample data, so establishing that relation requires dedicated measurements.

\section{B. Bi-2223 Double Pancake}

Application of a field independent $\alpha^{*}$ value as suggested by the short sample measurements on conductor B immediately leads to a reasonable fit. As for Coil A, the calculation is slightly 


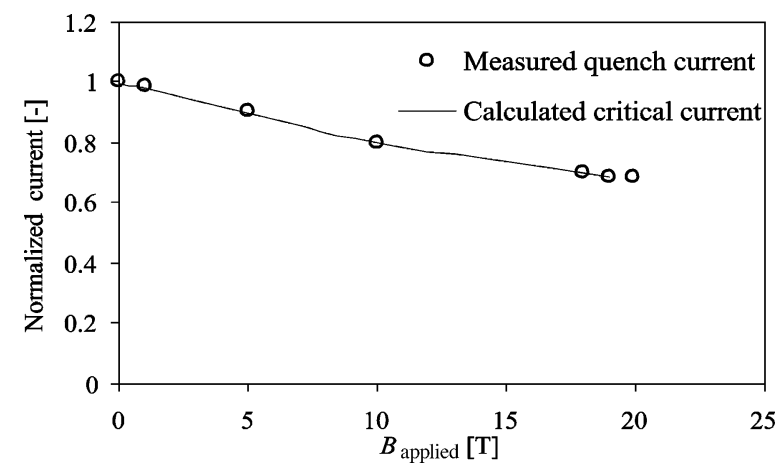

Fig. 7. Measured quench current and calculated $I_{\mathrm{C}}$ of an insert coil from conductor A.

TABLE IV

COIL B FIT PARAMETERS

\begin{tabular}{llll}
\hline \hline Current density & $I_{\mathrm{C} \_S S}($ self $)$ & $\alpha^{*}$ & $n$-value \\
\hline Axially variable & 312 & 2.7 & 20 \\
\hline
\end{tabular}

TABLE V

GEOMETRIC PARAMETERS FOR DOUBLE-PANCAKE STACKS

\begin{tabular}{lcccc}
\hline \hline & unit & Section A & Section B & Section C \\
\hline Inner diameter & $\mathrm{mm}$ & 41 & 106 & 156 \\
Outer diameter & $\mathrm{mm}$ & 96 & 146 & 165 \\
Height & $\mathrm{mm}$ & 184 & 184 & 209 \\
Turns [-] & - & 3723 & 2611 & 532 \\
\hline
\end{tabular}

below the measured values in the range of 5 to $15 \mathrm{~T}$. The calculated value for $I_{\text {C_SS }}($ self $), 312 \mathrm{~A}$, is significantly below measured values of around $408 \mathrm{~A}$.

\section{Double Pancake Stack}

A larger insert coil consisting of two stacks of 17 double pancakes each and surrounded by a layer wound section is constructed using conductor similar to conductor A. Key parameters are summarized in Table V, while a detailed description is provided elsewhere [3], [7].

The model is adapted by changing the segment dimensions to $3 \mathrm{~mm}$ radially and $11 \mathrm{~mm}$ axially. The latter corresponds to the total thickness of a double pancake unit in the stacks. Axial grading of $J$ is replaced by a varying conductor $I_{\mathrm{C} \_S S}$ (self) and $n$-value per unit, to reflect the variation of properties between conductor batches. These values are derived from properties of double pancakes as measured in individual tests at $4.2 \mathrm{~K}$.
This insert coil quenched before reaching the $10^{-4} \mathrm{~V} / \mathrm{m}$ criterion, so a direct comparison with the model is not possible, although the $I-V$ data does extrapolate to within a few amperes of the predicted $I_{\mathrm{c}}$. When both the quench current (157.2 A) and calculated critical current (207 A) at self-field are normalized, the model is found to describe accurately the relative change of insert $I_{\mathrm{C}}$ with background field.

\section{CONCLUSION}

The field dependence of the critical current in a Bi-2212 and a Bi-2223 double pancake can be modeled based on the measured field dependence of $I_{\mathrm{c}}$ of corresponding short samples. An axial variation of the current density in double pancakes is introduced to allow better modeling of the effects of field-angle differences between the midplane and top/bottom surfaces on the local critical current density. Anisotropy is modeled through the average grain misalignment $\alpha^{*}$. For the studied Bi-2223 conductor a constant value $\alpha^{*}$ is successfully used, as opposed to the $\mathrm{Bi}-2212$ case where the best results are obtained using a field-dependent value of $\alpha^{*}$. The latter is unexpected and warrants further investigation. The model is also accurate in describing the relative change of $I_{\mathrm{c}}$ with background field for a much larger insert. Predicting the self-field $I_{\mathrm{c}}$ for double pancakes based solely on short sample data, however, remains unsatisfactory.

\section{REFERENCES}

[1] K. R. Marken, H. Miao, B. Czabaj, and S. Hong, "BSCCO conductor development at Oxford superconducting technology," IEEE Trans. Appl. Supercond., vol. 13, pp. 3335-3338, 2003.

[2] M. Okada, "Development of Bi-2212/Ag round-shaped wire and magnet application," Supercond. Sci. Technol., vol. 13, pp. 29-33, 2000.

[3] H. W. Weijers, U. P. Trociewitz, K. Marken, M. Meinesz, H. Miao, and J. Schwartz, "The generation of 25.05 $\mathrm{T}$ using a $5.11 \mathrm{~T} \mathrm{Bi}_{2} \mathrm{Sr}_{2} \mathrm{CaCu}_{2} \mathrm{O}_{\mathrm{x}}$ superconducting insert magnet," Supercond. Sci. Technol., vol. 17, pp. 636-644, 2004.

[4] F. Hornung, M. Kläser, H. Leibrock, H. Müller, and T. Schneider, "Suitability of Bi-HTS wires for high field magnets," Physica C, vol. 401, pp. 218-221, 2004.

[5] O. van der Meer, B. ten Haken, and H. H. J. ten Kate, "A model to describe the angular dependence of the critical current in a Bi-2223/Ag superconducting tape," Physica C, vol. 357-360, pp. 1174-1177, 2001.

[6] H. W. Weijers, J. Schwartz, B. ten Haken, and H. H. J. ten Kate, "Effects of conductor anisotropy on the design of $\mathrm{Bi}-\mathrm{Sr}-\mathrm{Ca}-\mathrm{Cu}-\mathrm{O}$ sections of 25 T solenoids," Supercond. Sci. Technol., vol. 16, pp. 672-681, 2002.

[7] H. W. Weijers, U. P. Trociewitz, F. Trillaud, A. Mbaruku, P. V. S. S. Sastry, Y. S. Hascicek, J. Schwartz, K. Marken, M. Meinesz, and H. Miao, "Development of a 5 T HTS insert magnet as part of 25 T class magnets," IEEE Trans. Appl. Supercond., vol. 13, pp. 1396-1399, 2003.

[8] H. W. Weijers, B. ten Haken, H. H. J. ten Kate, and J. Schwartz, "Critical currents in Bi-Sr-Ca-Cu-O superconductors up to $33 \mathrm{~T}$ at $4.2 \mathrm{~K}$," IEEE Trans. Appl. Supercond., vol. 11, pp. 3956-3959, 2001. 